\title{
Synchronous Presentation of Ductal Carcinoma In Situ of the Breast with Follicular Lymphoma
}

\author{
Eun Young Kim, Sung-Im Do ${ }^{1}$, Yong Lai Park, Chan Heun Park \\ Departments of Surgery and ${ }^{1}$ Pathology, Kangbuk Samsung Hospital, Sungkyunkwan University School of Medicine, Seoul, Korea
}

A synchronous incidence of breast cancer and follicular lymphoma ( $\mathrm{FL})$ is uncommon. The association between these two conditions could be explained by the antigenic stimulation of the adjacent carcinoma and common genetic background. This study aimed to review the literature, and discuss the etiology and management of synchronous breast cancer and FL. Herein, we presented a case of synchronous ductal carcinoma in situ (DCIS) of the breast and FL involving multiple lymph nodes, including those in both axilla. A 49-year-old woman presented with palpable lumps in both axilla. She underwent lumpectomy, radiotherapy, and hormonal therapy for DCIS, while adjuvant chemotherapy was recommended for the lymphoma. Diagnosis was based on histopathologic analysis, such as excision or biopsy.

Key Words: Breast, Carcinoma, Lymphoma

\section{INTRODUCTION}

Breast cancer is the most common malignancy in women, and ductal carcinoma in situ (DCIS) is considered as a precursor of breast cancer [1]. Follicular lymphoma (FL) is the second most common form of non-Hodgkin lymphoma (NHL), which is a lymphoma of follicle center B cells. The frequency of FL in Asian countries is approximately $10 \%$ [2]. Previously, cases of breast cancer had been reported after the treatment of hematologic malignancies, such as Hodgkin's disease; however, the synchronous occurrence of breast cancer and lymphoma has been rarely reported [3]. Synchronous presentation of DCIS of the breast and FL is even more rare [4]. Herein, we presented a case where both pathologies coexisted without any history of previous therapy. In addition, a literature review was conducted, and the etiology and management of synchronous breast cancer and FL were discussed.

\section{CASE REPORT}

A 49-year-old woman presented with palpable lumps in both axilla a week prior to visiting the hospital. She had significant swelling on both axilla. No family history of breast cancer or lymphoma was re-

Correspondence: Chan Heun Park

Department of Surgery, Kangbuk Samsung Hospital, Sungkyunkwan University School of Medicine, 29 Saemunan-ro, Jongno-gu, Seoul 03181, Korea Tel: +82-2-2001-1730, Fax: +82-2-2001-1883, E-mail: chanheun1@gmail.com Received: Mar 22, 2017 Revised: Jul 6, 2017 Accepted:Oct 6, 2017 ported. Nonspecific induration was palpated on the upper inner quadrant of the right breast. Enlarged lymph nodes were observed in both axilla, which were correlated to swelling. Results of the physical examinations of the left breast, neck, abdomen, and both inguinal area were not significant.

Ultrasonography (US) (Philips Medical Systems, Bothell, USA) examination revealed diffused multifocal segmental duct dilatation with hypoechogenicity in the upper inner quadrant of the right breast (Figure 1A) and multiple enlarged lymph nodes with severe cortical thickening in both axilla (Figure 1B). The breast lesion was classified as C4b based on Breast Imaging Reporting and Data System.

US-guided core needle biopsy (CNB) of the right breast lesion revealed intraductal papilloma. Fine-needle aspiration biopsy of both axillary lymph nodes showed scattered lymphoid cells, and CNB was subsequently conducted for tissue diagnosis. In addition, $\mathrm{CNB}$ of the enlarged lymph nodes in the right axilla revealed atypical lymphoid hyperplasia. She underwent excisional biopsies of both the right breast lesion and axillary lymph node.

A histopathological examination of the right breast lesion showed intermediate nuclear grade DCIS with a solid and cribriform architectural pattern, which was $0.5 \times 0.4 \times 0.4 \mathrm{~cm}$ in size (Figure $1 C$ ). The right axillary lymph node was a low-grade FL (Figure 1D-F), which was positive for cluster of differentiation 20 (CD20), CD10, CD3, B-cell lymphoma 2 (BCL-2) protein, and BCL-6 protein, but negative for cyclin-D1. Ki-67 was 5\% (Figure 2). The lymph node was classified as 

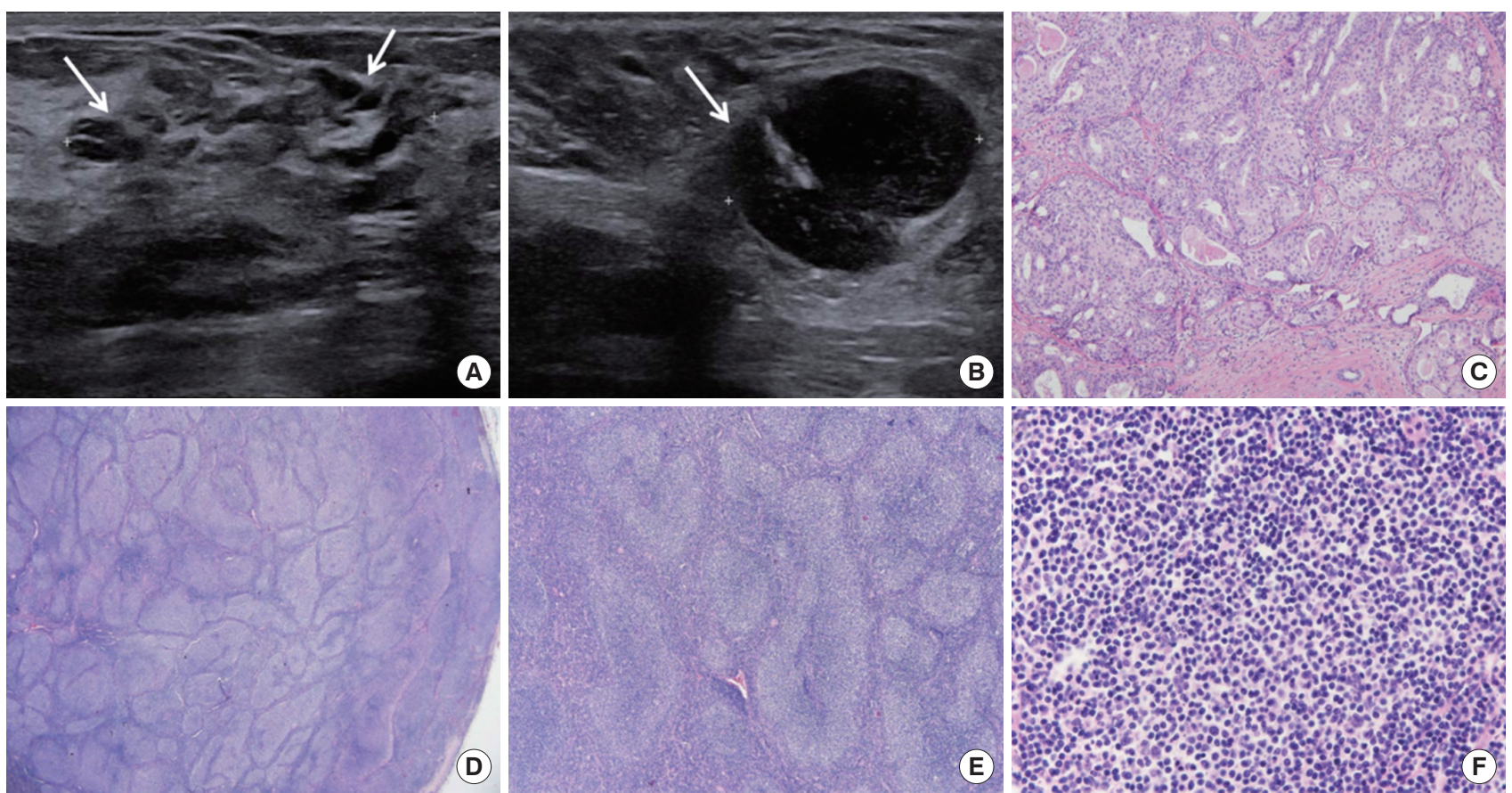

Figure 1. Ultrasonographic and histologic images of ductal carcinoma in situ (DCIS) and follicular lymphoma. (A) Ultrasonographic image shows a diffused multifocal segmental dilatation with hypoechogenic parenchymal pattern in the upper inner quadrant of the breast, which is $3.6 \times 1.0 \mathrm{~cm}$ in size (arrows). (B) Multiple enlarged lymph nodes are accompanied with severe cortical thickening in the right axilla (arrow). (C) Hematoxylin and eosin (H\&E) staining showed intermediate nuclear grade DCIS of the breast, which is composed of cells with mild to moderate variability in size and shape. The lesion had a cribriform and solid pattern $(\times 100)$. (D) H\&E staining depicted follicular lymphoma in the axilla $(\times 1.25)$. (E) H\&E staining shows that the neoplastic follicles are closely packed and lack a mantle zone ( $\times 40)$. (F) H\&E staining depicts the monotonous population of small cells with irregular nuclei and large cells that are rare $(\times 200)$.
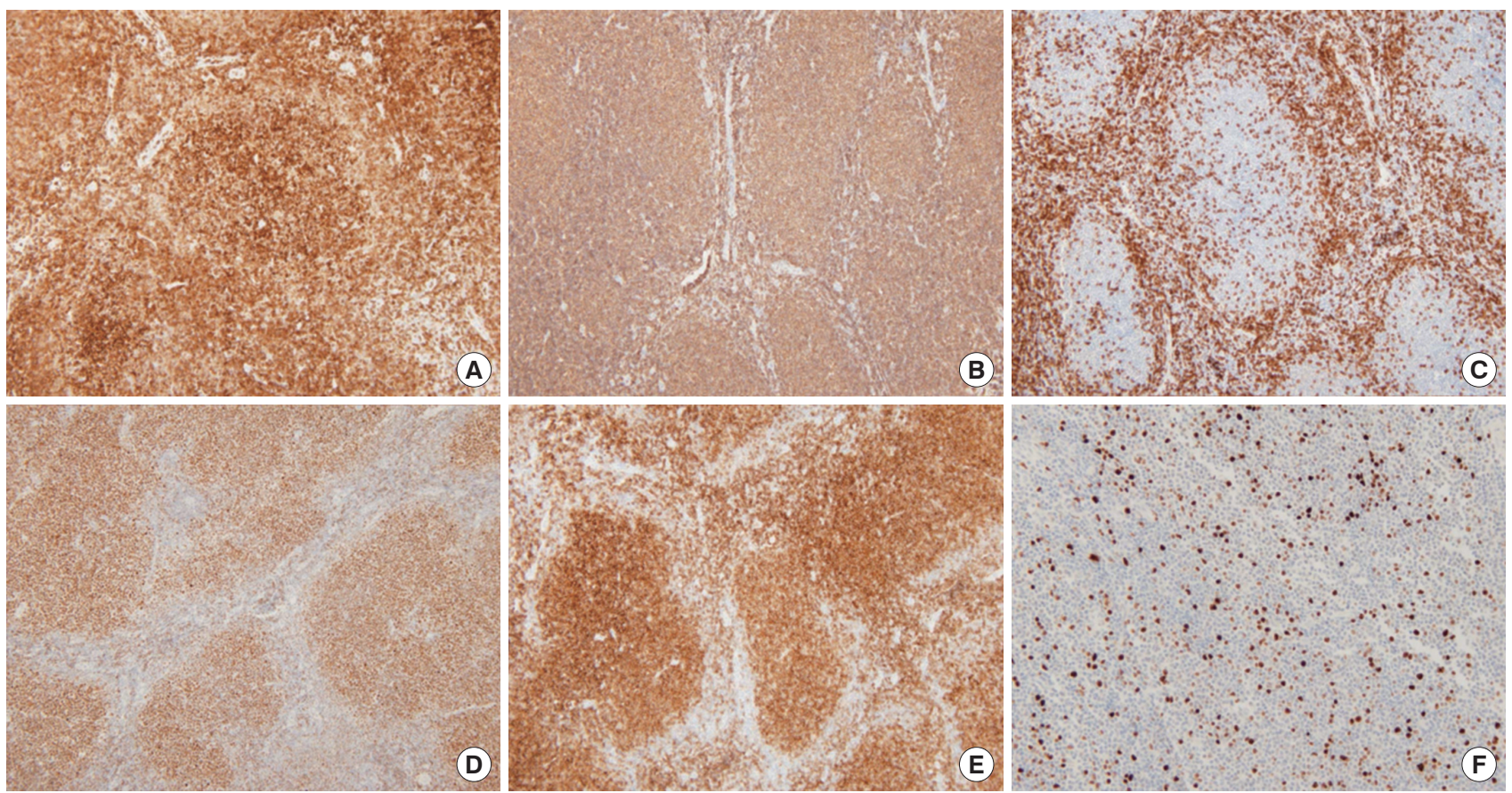

Figure 2. Immunohistochemistry staining results of follicular lymphoma $(\times 100)$. (A) The follicles are uniformly positive for B-cell lymphoma 2 (BCL-2). (B) The follicles and interfollicular regions contain $\mathrm{CD} 20^{+} \mathrm{B}$ cells. (C) CD3 shows remnant T cells. (D) BCL-6 is positive for follicles but to a less degree by interfollicular neoplastic cells. (E) CD10 expression is similar to BCL-6 expression. (F) Ki-67 is evenly expressed among the follicles and interfollicular regions. 

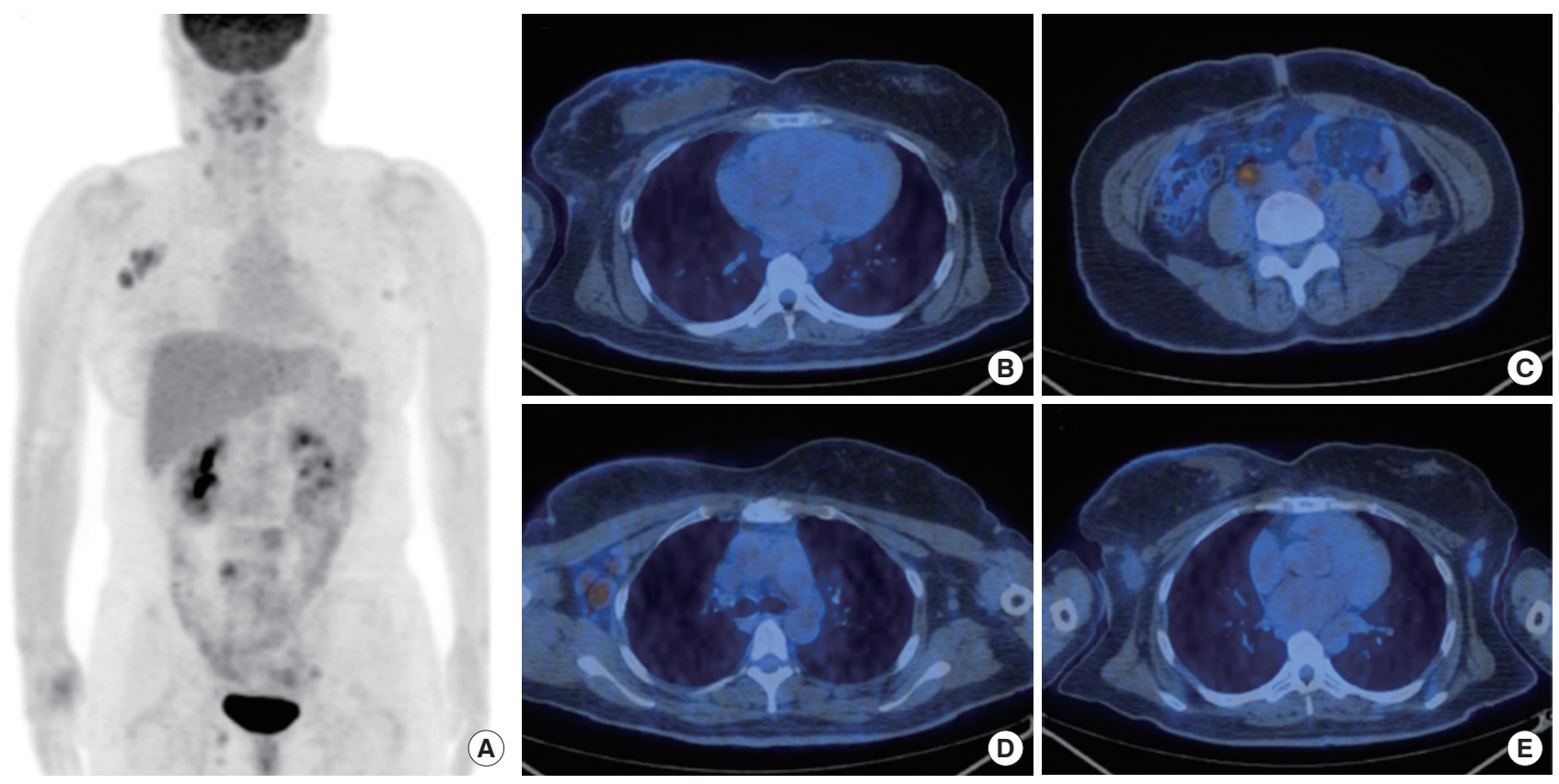

A)
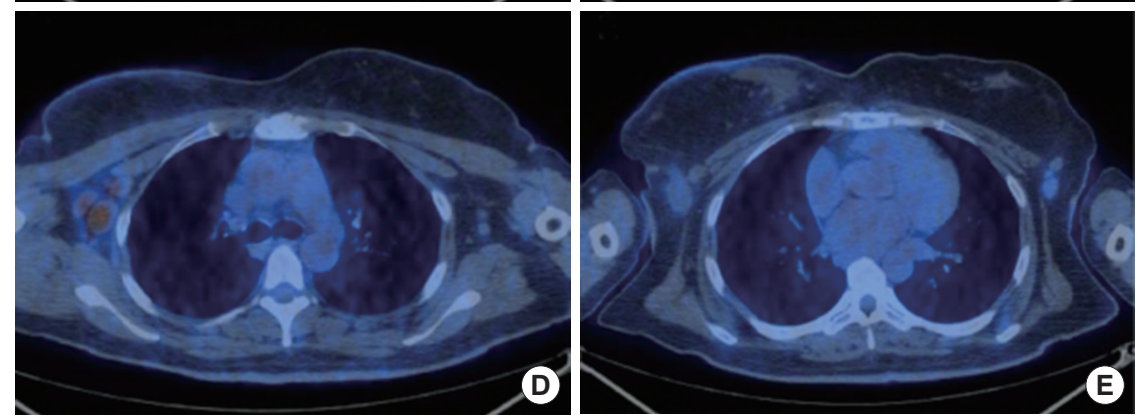

Figure 3. Images of positron emission tomography/computed tomography scans. (A) Maximal intensity projection images. (B) Mildly increased ${ }^{18} \mathrm{~F}$-fludeoxyglucose $\left({ }^{18} \mathrm{~F}-\mathrm{FDG}\right)$ uptake in the right breast mass (SUVmax, 2.2). (C) Increased ${ }^{18} \mathrm{~F}$-FDG uptake in the intra-abdominal lymph nodes along the right common iliac vessel (SUVmax, 4.3). (D) Increased ${ }^{18} \mathrm{~F}$-FDG uptake in the right axilla (SUVmax, 4.7). (E) Increased ${ }^{18} \mathrm{~F}$-FDG uptake in the left axilla (SUVmax, 1.7). SUVmax=maximum standardized uptake value.

stage III based on the American Joint Committee on Cancer (AJCC).

Positron emission tomography/computed tomography (PET/CT) (Discovery ST or STE PET/CT; General Electric Medical Systems, Milwaukee, USA) scans showed mild ${ }^{18}$ F-fluorodeoxyglucose $\left({ }^{18} \mathrm{~F}-\mathrm{FDG}\right)$ uptake in the right breast as well as in both axillary lymph nodes, right supraclavicular area, left upper back area, and intra-abdominal lymph nodes along the right common iliac vessel (Figure 3).

She underwent lumpectomy. Histopathologic evaluation of the breast specimen was also performed, and all margins were negative for cancer. Immunohistochemical staining of the DCIS was positive for estrogen receptor (Allred score, 7), progesterone receptor (Allred score, 7), and human epidermal growth factor receptor 2 protein expression. The AJCC stage was pTispN0M0, stage 0 .

Her local therapy was completed along with radiation therapy (RT) of the right breast, and adjuvant hormonal therapy (tamoxifen) was continued. She was scheduled for six cycles of chemotherapy consisting of cyclophosphamide, vincristine, adriamycin, and prednisone.

\section{DISCUSSION}

An increased risk of breast cancer has been reported in patients with lymphoma who have been treated with RT [3]. Few articles about secondary lymphoma after adjuvant RT or chemotherapy for breast cancer are also available [5]. However, these two diseases rarely coexist. Two or more primary carcinomas can coexist upon diagnosis or develop consequently, occasionally years after the resection of the first primary tumor. According to the Surveillance, Epidemiology, and End Results cancer registry, women with a history of breast cancer have a $12 \%$ reduced risk of developing NHL [6].

Several studies on the coexistence of DCIS and FL and its etiology are available. Suresh et al. [7] presented a case of breast cancer that coexisted with B-cell FL and discussed that the antigenic stimulation of the adjacent carcinoma, such as that in breast cancer, may have induced lymphoma genesis. This mechanism is similar to the role of Helicobacter pylori on inflammatory response, which causes stomach mucosa-associated lymphoid tissue (MALT) lymphoma. Stomach MALT lymphoma may be caused by continuous antigenic stimulation from H. pylori infection [8].

The association between these two diseases could also be explained by a common genetic background or the effects of epidemiologic factors. Ataxia telangiectasia mutated genes, which are observed in lymphoid and breast neoplasms, could be another factor [4]. Wiernik et al. 
[9] proposed that the mouse mammary tumor virus (MMTV)-like Env gene can be another cause. These gene sequences were detected in more than one-third of the breast cancer study population, and these sequences were also found in two patients with simultaneous presentation of breast cancer and lymphoma. Moreover, both breast cancer and lymphoma may be caused by an MMTV-like retrovirus.

Finally, even though it was not clear which malignancy-lymphoma or breast cancer - developed first, primary cancer-related immunological impairment predisposes secondary malignancies [10]. Lymphoma may block the flow in the lymphatic vessels and subsequently alter the pathway of metastasis in patients with breast cancer. Furthermore, neoplastic lymphoid cells could decrease interleukin-induced adhesion or the tissue necrosis factor of breast cancer cells to the endothelial layer of the axillary lymph nodes, promoting metastasis to certain lymph nodes [11].

In summary, the synchronous presentation of DCIS and FL is rare and difficult to diagnose. As in our case, the diagnosis of lymphadenopathy as a lymphoproliferative disease rather than breast metastasis was challenging. Multiple enlarged axillary lymph nodes on both sides that are found during physical examination or imaging studies should be meticulously diagnosed, particularly in patients with no signs of abnormal breast lesions. Such lymphadenopathy could prevent other malignancies or inflammatory disorders rather than metastatic diseases. Histopathologic confirmation of the synchronous presentation of both breast cancer and axillary lesions is indicated. Thus, clinicians must acknowledge that two different malignancies can coexist, and the recognition of the coexistence can help in the development of treatment plans for both diseases in the future.

\section{CONFLICT OF INTEREST}

The authors declare that they have no competing interests.

\section{REFERENCES}

1. Isaacs C, Stearns V, Hayes DF. New prognostic factors for breast cancer recurrence. Semin Oncol 2001;28:53-67.

2. Anderson JR, Armitage JO, Weisenburger DD. Epidemiology of the non-Hodgkin's lymphomas: distributions of the major subtypes differ by geographic locations. Non-Hodgkin's Lymphoma Classification Project. Ann Oncol 1998;9:717-20.

3. Travis LB, Hill D, Dores GM, Gospodarowicz M, van Leeuwen FE, Holowaty E, et al. Cumulative absolute breast cancer risk for young women treated for Hodgkin lymphoma. J Natl Cancer Inst 2005;97: 1428-37.

4. Cuff KE, Dettrick AJ, Chern B. Synchronous breast cancer and lymphoma: a case series and a review of the literature. J Clin Pathol 2010;63:555-7.

5. Lee KD, Chen SC, Chan CH, Lu CH, Chen CC, Lin JT, et al. Increased risk for second primary malignancies in women with breast cancer diagnosed at young age: a population-based study in Taiwan. Cancer Epidemiol Biomarkers Prev 2008;17:2647-55.

6. Curtis RE, Freedman DM, Ron E, Ries LA, Hacker DG, Edwards BK, et al. New Malignancies among Cancer Survivors: SEER Cancer Registries, 1973-2000. Bethesda: National Cancer Institute; 2006.

7. Suresh AV, Dadhich HK, Rao CR, Bapsy P, Batra U, Anupama G, et al. A case of breast cancer coexisting with B-cell follicular lymphoma. Austral Asian J Cancer 2007;6:155-6.

8. Susnik B, Jordi Rowe J, Redlich PN, Chitambar C, Chang CC, Kampalath B. A unique collision tumor in breast: invasive ductal carcinoma and mucosa-associated lymphoid tissue lymphoma. Arch Pathol Lab Med 2004;128:99-101.

9. Wiernik PH, Hu X, Ratech H, Fineberg S, Marino P, Schleider MA, et al. Non-Hodgkin's lymphoma in women with breast cancer. Cancer J 2000;6:336-42.

10. Ambrosiani L, Bellone S, Declich P, Tavani E, Pavia GF. Breast carcinoma metastatic to a lymphoma: report of a case with review of literature. Histopathology 1999;34:87-9.

11. Cohen PL, Brooks JJ. Lymphomas of the breast: a clinicopathologic and immunohistochemical study of primary and secondary cases. Cancer 1991;67:1359-69. 